\title{
Myocardial function and structure in aortic valve disease before and after surgery
}

\author{
Krayenbuehl, H P ; Schneider, J ; Turina, M ; Senning, A
}

\begin{abstract}
Left ventricular (LV) micromanometry, cine-angiography and endomyocardial biopsies were performed in 13 patients with aortic valve disease AVD) before and 12 to 28 months after successful valve replacement. (AVR). Patients with coronary artery disease were excluded. In nine patients (Group I: five $\mathrm{AS}$, four AI) postoperative LV ejection fraction (EF) and total pressure Vmax were normal(EF 0.61; Vmax $1.50 \mathrm{ML} / \mathrm{s}$ ). In four patients (Group II: three AS, one AT) postoperative EF (0.41) and Vmax $(1.21 \mathrm{ML} / \mathrm{s})$ were depressed. Pre-operative muscle fiber diameter (MFD; normal $<20 \mathrm{n}$ ) was 31 in Group I and 38 in Group II $(\mathrm{P}<0.01)$. After AVR MFD decreased to 27 in Group I $(\mathrm{P}<0.005)$ and to 28 in Group II $(\mathrm{P}<0.02)$. Prior to surgery EF and Vmax showed no significant correlation with the $\mathrm{LV}$ fibrous content $(\mathrm{FC}$ in $\mathrm{g} / \mathrm{m} 2 ; \mathrm{FC}=$ interstitial fibrosis in percent $\times \mathrm{LV}$ angiographic muscle mass $/ 100)$ in the 13 patients with AVD. After AVR, however, $\mathrm{FC}$ was related inversely to $\mathrm{EF}(\mathrm{P}<0.01, \mathrm{r}=-0.69)$ and to $\operatorname{Vmax}(\mathrm{P}<0.025, \mathrm{r}=-0.63)$. It is concluded that: (1) in AVD massive pre-operative fiber hypertrophy heralds impaired postoperative LV function; (2) fiber hypertrophy regresses following AVR regardless of the-LV functional state, and (3) the content of fibrous tissue appears to be a determinant of postoperative LV function
\end{abstract}

DOI: https://doi.org/10.1093/eurheartj/3.suppl_a.149

Posted at the Zurich Open Repository and Archive, University of Zurich

ZORA URL: https://doi.org/10.5167/uzh-154115

Journal Article

Published Version

Originally published at:

Krayenbuehl, H P; Schneider, J; Turina, M; Senning, A (1982). Myocardial function and structure in aortic valve disease before and after surgery. European Heart Journal, 3(Suppl A):149-153.

DOI: https://doi.org/10.1093/eurheartj/3.suppl_a.149 
Reversibility 



\title{
Myocardial function and structure in aortic valve disease before and after surgery*
}

\author{
H. P. Krayenbuehl, J. Schneider, M. Turina and $\AA$. Senning \\ Medical Policlinic, Cardiology, Institute of Pathology and Surgical Clinic A, University Hospital, \\ Zürich, Switzerland
}

\begin{abstract}
KEY WORDS: Left ventricular function, aortic valve replacement, myocardial hypertrophy and fibrosis.
Left ventricular ( $L V$ ) micromanometry, cine-angiography and endomyocardial biopsies were performed in 13 patients with aortic valve disease (AVD) before and 12 to 28 months after successful valve replacement. (AVR). Patients with coronary artery disease were excluded. In nine patients (Group I: five AS, four AI) postoperative $L V$ ejection fraction $(E F)$ and total pressure Vmax were normal $(E F \geqslant 0.61 ; V \max \geqslant 1.50 \mathrm{ML} / \mathrm{s})$. In four patients (Group II: three AS, one AI) postoperative EF $(0.41)$ and Vmax $(1.21 \mathrm{ML} / \mathrm{s})$ were depressed. Pre-operative muscle fiber diameter (MFD; normal $\leqslant 20 \mu)$ was $31 \mu$ in Group I and $38 \mu$ in Group II $(\mathrm{P}<0.01)$. After AVR MFD decreased to $27 \mu$ in Group I $(\mathrm{P}<0.005)$ and to $28 \mu$ in Group II $(\mathrm{P}<0.02)$. Prior to surgery EF and $V$ max showed no significant correlation with the $L V$ fibrous content ( $F C$ in $\mathrm{g} / \mathrm{m}^{2} ; F C=$ interstitial fibrosis in percent $\times L V$ angiographic muscle mass/100) in the 13 patients with AVD. After AVR, however, FC was related inversely to $E F(\mathrm{P}<0.01, r=-0.69)$ and to Vmax $(\mathrm{P}<0.025, r=-0.63)$. It is concluded that: (I) in AVD massive pre-operative fiber hypertrophy heralds impaired postoperative LV function; (2) fiber hypertrophy regresses following $A V R$ regardless of the $L V$ functional state, and (3) the content of fibrous tissue appears to be a determinant of postoperative LV function.
\end{abstract}

Left ventricular (LV) myocardial hypertrophy is a fundamental adaptive process in patients with chronic pressure or volume overload from aortic valve disease. Following successful aortic valve replacement, pre-operatively depressed LV function generally improves and angiographic LV muscle mass decreases $^{[1-5]}$. There are, however, occasional patients with postoperative residual LV dysfunction despite good valve function ${ }^{[1-3,5,6]}$. The question then arises whether in these patients irreversible structural alterations of the myocardium have taken place and if so, whether there are pre-operative indicators of the unfavorable outcome. In order to gain insight into the relations between function and structure of the left ventricle before and after removal of the hemodynamic burden we have studied invasively 13 patients before and after valve replacement and have carried out endomyocardial biopsies at both catheterizations.

Requests for reprints to: H. P. Krayenbuehl, M.D., Medical Policlinic, Cardiology, University Hospital, CH-8091 Zürich, Switzerland.

- Supported by a grant from the Swiss National Science Foundation
Since this series is small the results are only preliminary.

\section{Material and methods}

Among the 13 patients with aortic valve disease were eight with pure or predominant aortic stenosis (AS) the mean aortic valve area amounting to 0.75 $\mathrm{cm}^{2}(0.45-1 \cdot 1)$. Five patients had a pure or predominant aortic insufficiency (AI) with a mean aortic regurgitation fraction of $0.57(0.43-0.80)$. In 11 of the 13 patients coronary artery disease was excluded by selective arteriography. In two patients both below 40 years of age coronary arteriography was, in the absence of anginal symptoms, not carried out. Fourteen patients with a functional systolic murmur $(n=8)$ or atypical chest pain $(n=6)$ served as controls. No biopsies were performed in these patients. LV high-fidelity pressure measurements and left ventriculography were carried out as reported previously ${ }^{(3,7)}$.

The 13 patients with aortic valve disease were re- 
catheterized 18.2 months (12-28) after successful aortic valve replacement. All patients were asked by letter for the consent to undergo a second catheterization. Surgery consisted in the implantation of a bioprosthesis either of the Carpentier-Edwards $(n=7)$ or the Angell-Shiley $(n=2)$ type or of a BjörkShiley valve $(n=4)$. The postoperative aortic pressure gradients were small $(3-10 \mathrm{~mm} \mathrm{Hg})$. Minimal aortic regurgitation was present in two patients.

The following hemodynamic measurements were carried out before and after valve replacement: total pressure Vmax and Vpm were calculated from the LV high-fidelity pressure curves ${ }^{[7]}$ and LV volumes, ejection fraction (EF) and muscle mass (LMMI) were determined from the RAO cine-angiograms ${ }^{[3]}$.

The LV endomyocardial biopsy specimens ${ }^{[8]}$ were fixed in glutaraldehyde, embedded in epon and evaluated by light-microscopy in semi-thin sections $(1 \mu)$ stained by methyleneblue-azur II. From several sections of two biopsies of each catheterization muscle fiber diameter (MFD) and interstitial fibrosis (IF) were determined using a mechanical-optical pen (MOP-AM 03, Kontron Messgeräte GmbH, Zürich). For determining MFD at least 100 single measurements were carried out. IF was estimated using the point counting system ${ }^{[8]}$. All intersection points of the counting grid overlying interstitial non-muscular tissue were referred to as interstitial fibrosis. This is somewhat incorrect; but since fibrous tissue is the largely predominant component of the interstitial space the term 'fibrosis' was used in this context. LV fibrous content (FC) was calculated as the product of IF in percent $x$ angiographic muscle mass $/ 100$. Normal values for morphometric measurements were obtained from necropsy specimens. Normal MFD is $\leqslant 20 \mu$ and normal interstitial tissue amounts to less than $5 \%$

\section{Results}

In the 13 patients with aortic valve disease LV peak systolic pressure decreased from 171 to $137 \mathrm{~mm} \mathrm{Hg}$ $(P<0.005)$ and $\mathrm{LV}$ end-diastolic pressure from 17 to $11 \mathrm{~mm} \mathrm{Hg}(P<0.05)$ following valve replacement. LV end-diastolic volume decreased from 175 to 114 $\mathrm{ml} / \mathrm{m}^{2}(P<0.005)$ and LMMI from 180 to $115 \mathrm{~g} / \mathrm{m}^{2}$ $(P<0.005)$. EF (56 and 61\%), Vmax $(1.57$ and 1.64 muscle lengths/s) and Vpm (1.24 and 1.30 muscle lengths/s) remained unchanged. MFD decreased from 33.1 to $27.5 \mu(P<0.001)$ and IF increased from 17 to $27 \%(P<0.05)$. FC remained unchanged $(28$ and $\left.32 \mathrm{~g} / \mathrm{m}^{2}\right)$.

For the analysis of the relationship between function and structure the patients were grouped accord- ing to the functional status after valve replacement. In Group I there were nine patients (five AS, four AI) with normal postoperative $\mathrm{LV}$ function as evaluated by the EF $(\geqslant 61 \%$ ) and total pressure $V \max$ $(\geqslant 1.50 \mathrm{ML} / \mathrm{s})$. In Group II there were four patients (three AS, one AI) with depressed postoperative LV function the $E F$ averaging 0.41 and $V \max 1.21 \mathrm{ML} / \mathrm{s}$. All except one of these patients had depressed LV

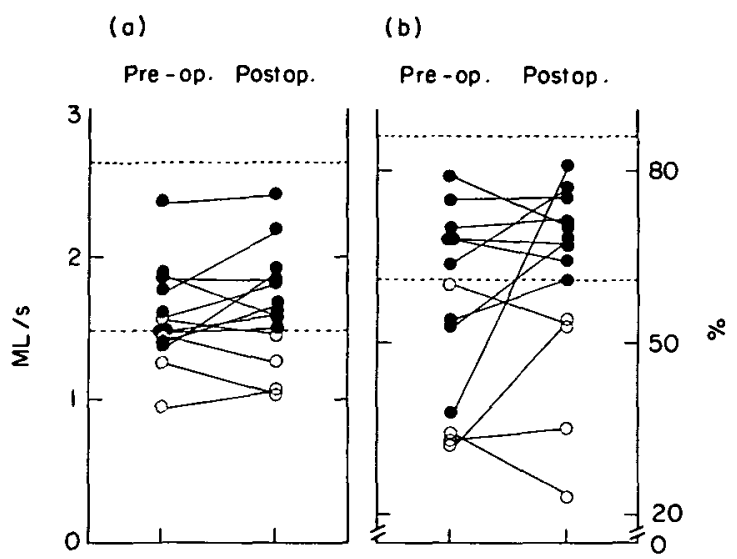

Figure I (a) Total pressure Vmax and (b) left ventricular monoplane (RAO) ejection fraction (EF) in 13 patients with aortic valve disease pre- and postoperatively. --- = Upper and lower limit of Vmax and EF respectively, determined in 14 control patients; $M L=$ muscle lengths; - = postoperative normal LV function (Group I, $n=9$ ); $O=$ postoperative depressed LV function (Group II, $n=4$ ).

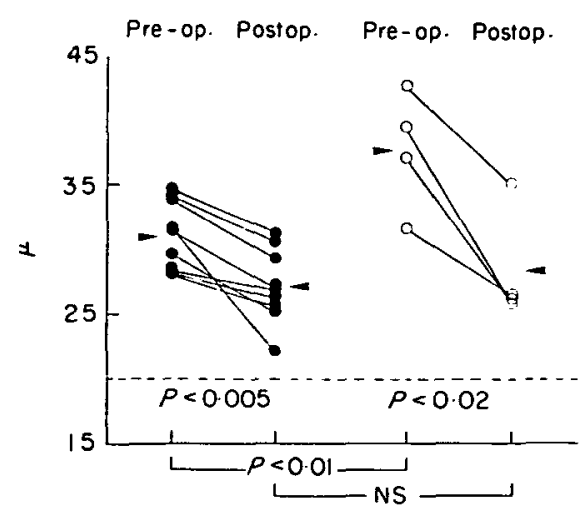

Figure 2 Muscle fiber diameter before and after surgery in nine patients with aortic valve disease and normal postoperative left ventricular function ( $=$ Group I) and in four patients with depressed postoperative contractile function $(\mathrm{O}=$ Group II). The dashed horizontal line indicates the upper limit of normal as determined in myocardial necropsy specimens from patients without heart disease. The arrows indicate the mean values. $\mathrm{NS}=$ not significant. 


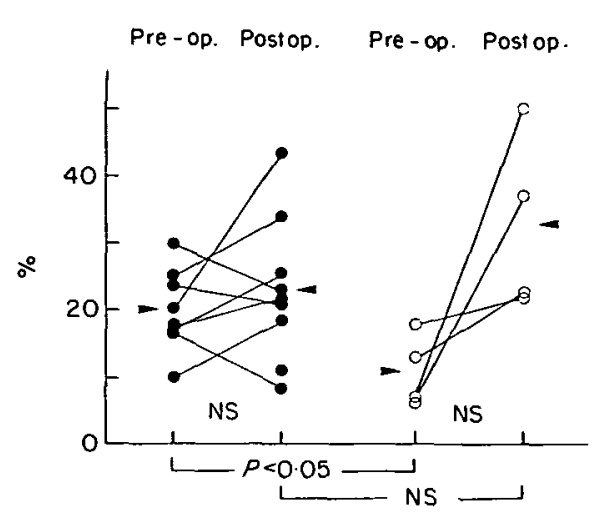

Figure 3 Interstitial fibrosis in nine patients with normal postoperative left ventrical function $(\bullet=$ Group I) and four patients with depressed postoperative contractile function $(\mathrm{O}=$ Group II). Abbreviations as in Fig. 2.

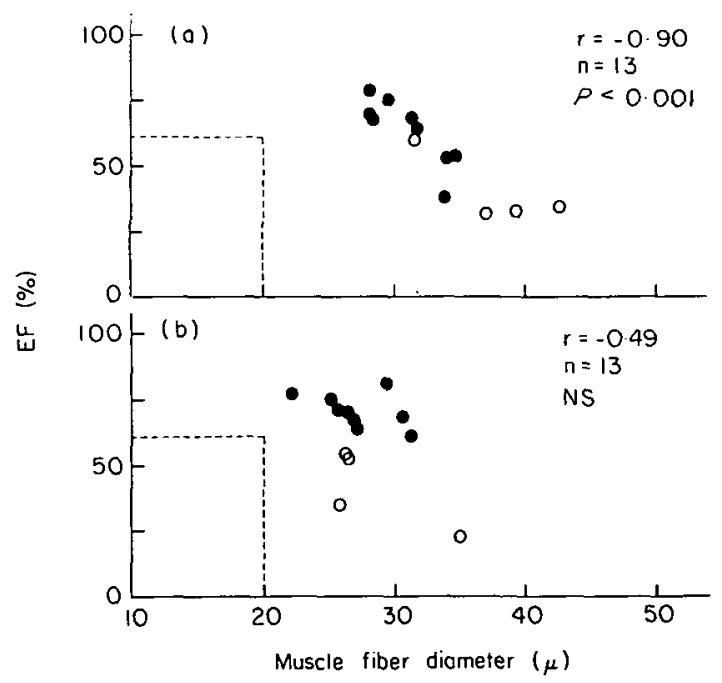

Figure 4 Correlation between left ventricular ejection fraction (EF) and muscle fiber diameter at the pre-operative (a) and the postoperative (b) investigation. = Group I; $\mathrm{O}=$ Group II; -- $=$ the lower limit of normal of the ejection fraction and the upper limit of muscle fiber diameter in normal necropsy specimens. Whereas there was a significant inverse relationship between EF and muscle fiber diameter before surgery EF and muscle fiber diameter did not correlate after valve replacement, $r=$ Correlation coefficient; $\mathbf{n}=$ number of patients; NS $=$ not significant.

function prior to surgery whereas in Group I only three of the nine patients had abnormal LV function at the pre-operative evaluation (Fig. 1). Following surgery MFD (Fig. 2) decreased significantly in both groups. There was no significant difference of the post-

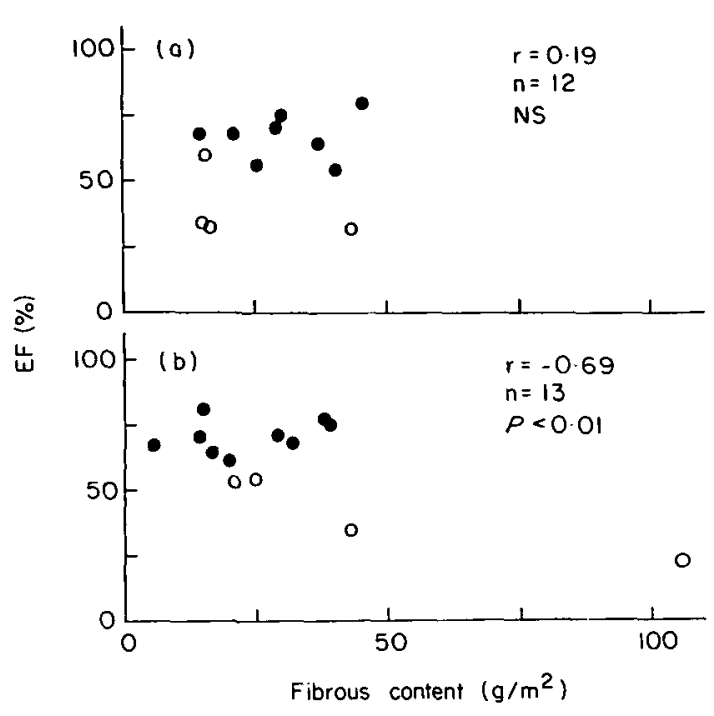

Figure 5 Correlation between left ventricular ejection fraction (EF) and fibrous content at the pre-operative (a) and the postoperative (b) investigation. Postoperatively there was a significant correlation between EF and fibrous content. This was not the case at the pre-operative study. Abbreviations as in Fig. 4.

operative fiber thickness in the two groups. In no instance did postoperative MFD reach the upper limit of normal $(20 \mu)$. The pre-operative MFD was significantly larger in the patients with an unfavorable postoperative result $(38 \mu)$ than in those with normal postoperative LV function (31 $\mu$ ). IF (Fig. 3). showed variable changes in Group I after surgery. In Group II IF increased in all instances; this increase was, however, not significant due to the large scatter in the extent of increase and the small number of observations. Astonishingly pre-operative IF was smaller in Group II than in Group I whereas after surgery it tended to be greater in Group II than in Group I. FC did not change significantly in the two groups following surgery.

At the pre-operative study there was a close inverse correlation ( $r=-0.90$ ) between EF and MFD (Fig. 4a) whereas at the postoperative study EF did not correlate with MFD (Fig. 4b). When EF was correlated to LVFC there was no significant correlation for the pre-operative data (Fig. 5a). In contrast, the postoperative data showed a significant inverse correlation between EF and FC (Fig 5b). There were no significant correlations between EF and IF, neither pre-operatively nor postoperatively.

The comparison of the individual changes of MFD with those of LMMI occurring following valve replacement (Fig. 6) showed that except in one case, reductions in fiber diameter were accompanied by 


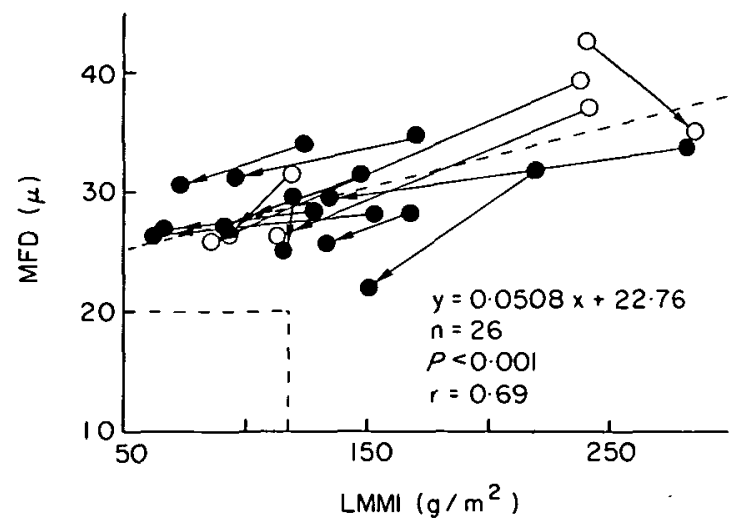

Figure 6 Correlation between individual muscle fiber diameter (MFD) and left ventricular angiographic mass (LMMI) before and after surgery. Except in one patient, reduction of MFD was associated with a decrease of LMMI. Note that despite normalization of the angiographic mass (decrease below $117 \mathrm{~g} / \mathrm{cm}^{2}$ ) in nine of 13 patients postoperative MFD remained above the upper limit of normal $(20 \mu)$ in all instances. The dashed quadrangle indicates the upper limits of normality of MFD and LMMI. The ascending dashed line is the regression line through all preand postoperative data points. Abbreviations as in Fig. 4.

reductions of angiographic mass. It is noteworthy, however, that despite normalization of the angiographic mass, i.e. a decrease below the upper limit of normality $\left(117 \mathrm{~g} / \mathrm{m}^{2}\right)$ in nine of 13 patients, postoperative myocardial fiber thickness remained above the upper limit of normal $(20 \mu)$ in all instances.

\section{Discussion}

Although generally left ventricular function improves considerably following aortic valve replacement in patients with pre-operatively depressed contractility ${ }^{[1-5.9]}$ there are patients with persistent LV dysfunction despite good prosthetic valve function $^{[1-3,5,6]}$. In these patients irreversible damage of the myocardium appears to exist and hence the 'myocardial factor' becomes the major determinant of the prognosis. In patients with aortic valve disease Schwarz and coworkers $^{[9]}$ have correlated preoperative LV function parameters with structural alterations evaluated from transmural LV biopsies which they performed at surgery. They found no significant relationship between the extent of interstitial myocardial fibrosis and pre-operative depression of $\mathrm{LV}$ function. The ultrastructural degenerative cell changes present in 13 of 15 patients were, however, felt to explain the pre-operative impairment of contractile function. Since LV ejection performance became normal six months after aortic valve replacement it was speculated that the ultrastructural myocardial changes are fully reversible.

More recently Schwarz and coworkers ${ }^{[10]}$ have confirmed the lack of correlation between preoperative EF and the amount of interstitial fibrosis in patients with AS. A reduction of myofibrils was found in the patients with decompensated aortic stenosis and advanced hypertrophy. The authors concluded that the deficit of myofibrils represents the 'myocardial factor' in chronic pressure overload. No data were presented which would support the concept that this deficit of myofibrils may also be responsible for continuing $\mathrm{LV}$ dysfunction late after aortic valve replacement.

In the present study we have found that in patients with aortic valve disease pre-operative interstitial fibrosis and fibrous content are not indicators of postoperative LV function. In contrast excessive preoperative fiber hypertrophy was shown to herald persistent impairment of left ventricular contractility. Whereas fiber diameter regressed to similar values in patients with normal and depressed postoperative LV function interstitial fibrosis increased in all four patients with compromised postoperative LV function. Hence one may speculate that reduction in size of excessively hypertrophied cells following removal of the hemodynamic burden is associated with an ongoing process of fibrosis which ultimately may determine postoperative functional impairment. In this respect it is noteworthy that at the postoperative evaluation EF was correlated inversely to fibrous content. Finally this study shows that postoperative normalization of angiographic mass as a measure of chamber hypertrophy does not necessarily imply a normalization of fiber thickness, i.e. hypertrophy at the cellular level.

\section{References}

[1] Kennedy JW, Doces J, Stewart DK. Left ventricular function before and following aortic valve replacement. Circulation 1977; 56: 944-50.

[2] Smith N, McAnulty JH, Rahimtoola SH. Severe aortic stenosis with impaired left ventricular function and clinical heart failure: Results of valve replacement. Circulation 1978; 58: 255-64

[3] Krayenbuehl HP, Turina M, Hess OM, Rothlin M, Senning $\AA$. Pre- and postoperative left ventricular contractile function in patients with aortic valve disease. Br Heart J 1979; 41: 204-13.

[4] Schwarz F, Flameng W, Langebartels F, Sesto M, Walter $P$, Schlepper $M$. Impaired left ventricular function in chronic aortic valve disease: Survival and function after replacement by Björk-Shiley prosthesis. Circulation 1979; 60: 48-58. 
[5] Clark DG, McAnulty JH, Rahimtoola SH. Valve replacement in aortic insufficiency with left ventricular dysfunction. Circulation 1980; 61: 411-21.

[6] Toussaint C, Cribier A, Cazor JL, Soyer R, Letac B. Hemodynamic and angiographic evaluation of aortic regurgitation eight and 27 months after aortic valve replacement. Circulation 1981; 64: 456-63.

[7] Krayenbuehl HP, Rutishauser W, Wirz P, Amende I, Mehmel H. High-fidelity left ventricular pressure measurements for the assessment of cardiac contractility in man. Am J Cardiol 1973; 31: 415-27.

[8] Hess OM, Schneider J, Koch R, Bamert C, Grimm J,
Krayenbuehl HP. Diastolic function and myocardial hypertrophy. Circulation 1981; 63: 360-71.

[9] Schwarz F, Flameng W, Schaper J, Langebartels F, Sesto M, Hehrlein F, Schlepper M. Myocardia structure and function in patients with aortic valve disease and their relation to postoperative results. Am J Cardiol 1978; 41: 661-9.

[10] Schwarz F, Schaper J, Kittstein D, Flameng W, Walter P, Schaper W. Reduced volume fraction of myofibrils in myocardium of patients with decompensated pressure overload. Circulation 1981; 63: 1299 304. 
